\title{
Positive solutions of Riemann-Stieltjes integral boundary problems for the nonlinear coupling system involving fractional-order differential
}

\author{
Kaihong Zhao* and Ping Gong
}

"Correspondence:

zhaokaihongs@126.com Department of Applied

Mathematics, Kunming University of Science and Technology, Kunming, Yunnan 650093, China

\begin{abstract}
In this article, we study Riemann-Stieltjes integral boundary value problems of nonlinear fractional functional differential coupling system involving higher-order Caputo fractional derivatives. Some sufficient criteria are obtained for the existence, multiplicity, and nonexistence of positive solutions by applying fixed-point theorems on a convex cone. As applications, some examples are provided to illustrate our main results.
\end{abstract}

Keywords: coupling fractional differential system; positive solutions; Riemann-Stieltjes integral BVPs; fixed point theorem

\section{Introduction}

Fractional differential equations arise in many engineering and scientific disciplines as the mathematical modeling of systems and processes in the fields of physics, chemistry, aerodynamics, electrodynamics of complex medium, polymer rheology, Bode's analysis of feedback amplifiers, capacitor theory, electrical circuits, electron-analytical chemistry, biology, control theory, fitting of experimental data, and so forth, and involves derivatives of fractional order. Fractional derivatives provide an excellent tool for the description of memory and hereditary properties of various materials and processes. This is the main advantage of fractional differential equations in comparison with classical integer-order models. In consequence, the subject of fractional differential equations is gaining much importance and attention. Especially, there have been many papers focused on boundary value problems of fractional ordinary differential equations (see [1-16]). Moreover, the boundary value problems with Riemann-Stieltjes integral boundary condition arise in a variety of different areas of applied mathematics and physics (for more comments on Stieltjes integral boundary condition and its importance, we refer the reader to the papers by Webb and Infante [11, 12] and their other related works). For example, blood flow problems, chemical engineering, thermo-elasticity, underground water flow, population dynamics, and so on can be reduced to nonlocal integral boundary problems. Nonlocal boundary value problems of fractional-order differential equations constitute a class of very interesting and important problems. This type of boundary value problems has been

○2014 Zhao and Gong; licensee Springer. This is an Open Access article distributed under the terms of the Creative Commons Attribution License (http://creativecommons.org/licenses/by/2.0), which permits unrestricted use, distribution, and reproduction in any medium, provided the original work is properly cited. 
investigated in $[9,10,13-16]$. To the best of our knowledge, there are only few papers dealing with the existence, multiplicity, and nonexistence of positive solutions of RiemannStieltjes integral boundary problems for high-order nonlinear fractional differential coupling system. Therefore, we study the existence, multiplicity, and nonexistence of positive solutions for the following high-order nonlinear fractional differential coupling system (abbreviated by BVPs (1.1)-(1.2) throughout this paper):

$$
\begin{cases}D_{0+}^{\alpha} u(t)+f\left(t, u(t), v(t), u^{\prime}(t), v^{\prime}(t)\right)=0, & t \in(0,1), n-1<\alpha \leq n, \\ D_{0+}^{\beta} \nu(t)+g\left(t, u(t), u^{\prime}(t)\right)=0, & t \in(0,1), m-1<\beta \leq m,\end{cases}
$$

subject to the integral boundary conditions

$$
\begin{cases}u(0)=u^{\prime \prime}(0)=\cdots=u^{(n-1)}(0)=0, & u^{\prime}(1)=\int_{0}^{1} u(s) d H(s), \\ v(0)=v^{\prime \prime}(0)=\cdots=v^{(m-1)}(0)=0, & v^{\prime}(1)=\int_{0}^{1} v(s) d K(s),\end{cases}
$$

where $n, m \in \mathbb{N}, n, m \geq 3 . D_{0+}^{\alpha}, D_{0+}^{\beta}$ are the Caputo fractional derivatives of order $n-1<$ $\alpha \leq n, m-1<\beta \leq m . f:[0,1] \times[0,+\infty)^{4} \rightarrow[0,+\infty), g:[0,1] \times[0,+\infty)^{2} \rightarrow[0,+\infty)$ are continuous functions. The integrals from (1.2) are Riemann-Stieltjes integrals. $H, K$ : $[0,1] \rightarrow \mathbb{R}$ are the function of bounded variation with $\Delta_{1} \triangleq 1-\int_{0}^{1} s d H(s) \neq 0$ and $\Delta_{2} \triangleq$ $1-\int_{0}^{1} s d K(s) \neq 0$. To the best of our knowledge, the study of existence of positive solutions of nonlinear fractional differential system (1.1)-(1.2) has not been done.

The rest of this paper is organized as follows. In Section 2, we recall some useful definitions and properties, and present the properties of the Green's functions. In Section 3, we give some sufficient conditions for the existence and nonexistence of positive solutions for boundary value problem (1.1)-(1.2). Some examples are also provided to illustrate our main results in Section 4.

\section{Preliminaries}

For the convenience of the reader, we present here the necessary definitions from fractional calculus theory. These definitions and properties can be found in the recent literature.

Definition 2.1 (see $[17,18]$ ) The Riemann-Liouville fractional integral of order $\alpha>0$ of a function $f:(0, \infty) \rightarrow \mathbb{R}$ is given by

$$
I_{0+}^{\alpha} f(t)=\frac{1}{\Gamma(\alpha)} \int_{0}^{t}(t-s)^{\alpha-1} f(s) d s
$$

provided that the right-hand side is pointwise defined on $(0, \infty)$.

Definition 2.2 (see $[17,18]$ ) The Caputo fractional derivative of order $\alpha>0$ of a continuous function $f:(0, \infty) \rightarrow \mathbb{R}$ is given by

$$
D_{0+}^{\alpha} f(t)=\frac{1}{\Gamma(n-\alpha)} \int_{0}^{t} \frac{f^{(n)}(s)}{(t-s)^{\alpha-n+1}} d s
$$

where $n-1<\alpha \leq n$, provided that the right-hand side is pointwise defined on $(0, \infty)$. 
Lemma 2.1 (see [17]) Assume that $u \in C(0,1) \cap L(0,1)$ with a Caputo fractional derivative of order $\alpha>0$ that belongs to $u \in C^{n}[0,1]$, then

$$
I_{0+}^{\alpha} D_{0+}^{\alpha} u(t)=u(t)+C_{0}+C_{1} t+\cdots+C_{n-1} t^{n-1},
$$

for some $C_{i} \in \mathbb{R}, i=0,1, \ldots, n-1$, where $n$ is the smallest integer greater than or equal to $\alpha$.

Here we introduce the following useful fixed-point theorems.

Lemma 2.2 (see [19]) Let E be a Banach space, $P \subseteq E$ a cone, and $\Omega_{1}, \Omega_{2}$ are two bounded open balls of $E$ centered at the origin with $0 \in \Omega_{1}$ and $\bar{\Omega}_{1} \subset \Omega_{2}$. Suppose that $T: P \cap\left(\bar{\Omega}_{2} \backslash\right.$ $\left.\Omega_{1}\right) \rightarrow P$ is a completely continuous operator such that either

(i) $\|T u\| \leq\|u\|, u \in P \cap \partial \Omega_{1}$ and $\|T u\| \geq\|u\|, u \in P \cap \partial \Omega_{2}$, or

(ii) $\|T u\| \geq\|u\|, u \in P \cap \partial \Omega_{1}$ and $\|T u\| \leq\|u\|, u \in P \cap \partial \Omega_{2}$

holds. Then $T$ has at least one fixed point in $P \cap\left(\bar{\Omega}_{2} \backslash \Omega_{1}\right)$.

Let $E$ be a real Banach space with a cone $P \subset E$. Define a partial order $\prec$ in $E$ as $v \prec u$ if $u-v \in P$. For $u-v \in E$, the order interval $\langle v, u\rangle$ is defined as $\langle v, u\rangle=\{x \in E: v \prec x \prec u\}$.

Lemma 2.3 (see [20]) Let $P$ be a normal cone in a real Banach space $E,\left\langle v_{0}, u_{0}\right\rangle \subset P$ and $T:\left\langle v_{0}, u_{0}\right\rangle \rightarrow\left\langle v_{0}, u_{0}\right\rangle$ be an increasing operator. If $T$ is completely continuous, then $T$ has a fixed point $u^{*} \in\left\langle v_{0}, u_{0}\right\rangle$.

Now we present the Green's functions for system associated with BVPs (1.1)-(1.2).

Lemma 2.4 If $H:[0,1] \rightarrow \mathbb{R}$ is a function of bounded variation with $\Delta_{1} \triangleq 1-\int_{0}^{1} s d H(s) \neq 0$ and $y \in C([0,1])$, then the unique solution of

$$
\left\{\begin{array}{l}
D_{0^{+}}^{\alpha} u(t)+y(t)=0, \quad t \in(0,1), n-1<\alpha \leq n, n \geq 3 \\
u(0)=u^{\prime \prime}(0)=\cdots=u^{(n-1)}(0)=0, \quad u^{\prime}(1)=\int_{0}^{1} u(s) d H(s),
\end{array}\right.
$$

is given by

$$
u(t)=\int_{0}^{1} G_{\alpha}(t, s) y(s) d s
$$

where

$$
G_{\alpha}(t, s)=g_{\alpha}(t, s)+\frac{t}{\Delta_{1}} \int_{0}^{1} g_{\alpha}(\tau, s) d H(\tau),
$$

and

$$
g_{\alpha}(t, s)= \begin{cases}\frac{(\alpha-1) t(1-s)^{\alpha-2}-(t-s)^{\alpha-1}}{\Gamma(\alpha)}, & 0 \leq s \leq t \leq 1, \\ \frac{(\alpha-1) t(1-s)^{\alpha-2}}{\Gamma(\alpha)}, & 0 \leq t \leq s \leq 1 .\end{cases}
$$

Proof Applying Lemma 2.1, Eq. (2.1) is translated into an equivalent integral equation

$$
u(t)=-\frac{1}{\Gamma(\alpha)} \int_{0}^{t}(t-s)^{\alpha-1} y(s) d s+C_{0}+C_{1} t+\cdots+C_{n-1} t^{n-1} .
$$


In the light of $u(0)=u^{\prime \prime}(0)=\cdots=u^{(n-1)}(0)=0$, we have $C_{0}=C_{2}=\cdots=C_{n-1}=0$. From $u^{\prime}(1)=\int_{0}^{1} u(s) d H(s)$, we deduce

$$
\begin{aligned}
- & \frac{1}{\Gamma(\alpha)} \int_{0}^{1}(\alpha-1)(1-s)^{\alpha-2} y(s) d s+C_{1} \\
\quad & \int_{0}^{1}\left[-\frac{1}{\Gamma(\alpha)} \int_{0}^{s}(s-\tau)^{\alpha-1} y(\tau) d \tau+C_{1} s\right] d H(s),
\end{aligned}
$$

namely,

$$
\begin{aligned}
& C_{1}\left(1-\int_{0}^{1} s d H(s)\right) \\
& \quad=\frac{\alpha-1}{\Gamma(\alpha)} \int_{0}^{1}(1-s)^{\alpha-2} y(s) d s-\frac{1}{\Gamma(\alpha)} \int_{0}^{1}\left(\int_{0}^{s}(s-\tau)^{\alpha-1} y(\tau) d \tau\right) d H(s),
\end{aligned}
$$

which implies

$$
\begin{aligned}
C_{1} & =\frac{\alpha-1}{\Delta_{1} \Gamma(\alpha)} \int_{0}^{1}(1-s)^{\alpha-2} y(s) d s-\frac{1}{\Delta_{1} \Gamma(\alpha)} \int_{0}^{1}\left(\int_{0}^{s}(s-\tau)^{\alpha-1} y(\tau) d \tau\right) d H(s) \\
& =\frac{\alpha-1}{\Delta_{1} \Gamma(\alpha)} \int_{0}^{1}(1-s)^{\alpha-2} y(s) d s-\frac{1}{\Delta_{1} \Gamma(\alpha)} \int_{0}^{1}\left(\int_{\tau}^{1}(s-\tau)^{\alpha-1} d H(s)\right) y(\tau) d \tau \\
& =\frac{\alpha-1}{\Delta_{1} \Gamma(\alpha)} \int_{0}^{1}(1-s)^{\alpha-2} y(s) d s-\frac{1}{\Delta_{1} \Gamma(\alpha)} \int_{0}^{1}\left(\int_{s}^{1}(\tau-s)^{\alpha-1} d H(\tau)\right) y(s) d s .
\end{aligned}
$$

Therefore, the solution of BVPs (2.1) is

$$
\begin{aligned}
u(t)= & -\frac{1}{\Gamma(\alpha)} \int_{0}^{t}(t-s)^{\alpha-1} y(s) d s+\frac{t}{\Delta_{1} \Gamma(\alpha)}\left[\int_{0}^{1}(\alpha-1)(1-s)^{\alpha-2} y(s) d s\right. \\
& \left.-\int_{0}^{1}\left(\int_{s}^{1}(\tau-s)^{\alpha-1} d H(\tau)\right) y(s) d s\right] \\
= & \frac{1}{\Gamma(\alpha)}\left\{\int_{0}^{t}\left[(\alpha-1) t(1-s)^{\alpha-2}-(t-s)^{\alpha-1}\right] y(s) d s+\int_{t}^{1}(\alpha-1) t(1-s)^{\alpha-2} y(s) d s\right. \\
& -\int_{0}^{1}(\alpha-1) t(1-s)^{\alpha-2} y(s) d s+\frac{t}{\Delta_{1}}\left[\int_{0}^{1}(\alpha-1)(1-s)^{\alpha-2} y(s) d s\right. \\
& \left.\left.-\int_{0}^{1}\left(\int_{s}^{1}(\tau-s)^{\alpha-1} d H(\tau)\right) y(s) d s\right]\right\} \\
= & \frac{1}{\Gamma(\alpha)}\left\{\int_{0}^{t}\left[(\alpha-1) t(1-s)^{\alpha-2}-(t-s)^{\alpha-1}\right] y(s) d s\right. \\
& +\int_{t}^{1}(\alpha-1) t(1-s)^{\alpha-2} y(s) d s-\frac{1}{\Delta_{1}}\left(1-\int_{0}^{1} \tau d H(\tau)\right) \int_{0}^{1}(\alpha-1) t(1-s)^{\alpha-2} y(s) d s \\
& \left.+\frac{t}{\Delta_{1}}\left[\int_{0}^{1}(\alpha-1)(1-s)^{\alpha-2} y(s) d s-\int_{0}^{1}\left(\int_{s}^{1}(\tau-s)^{\alpha-1} d H(\tau)\right) y(s) d s\right]\right\} \\
= & \frac{1}{\Gamma(\alpha)}\left\{\int_{0}^{t}\left[(\alpha-1) t(1-s)^{\alpha-2}-(t-s)^{\alpha-1}\right] y(s) d s+\int_{t}^{1}(\alpha-1) t(1-s)^{\alpha-2} y(s) d s\right. \\
& +\frac{t}{\Delta_{1}}\left[\int_{0}^{1}\left(\int_{0}^{1}(\alpha-1) \tau(1-s)^{\alpha-2} d H(\tau)\right) y(s) d s\right.
\end{aligned}
$$




$$
\begin{aligned}
& \left.\left.-\int_{0}^{1}\left(\int_{s}^{1}(\tau-s)^{\alpha-1} d H(\tau)\right) y(s) d s\right]\right\} \\
= & \frac{1}{\Gamma(\alpha)}\left\{\int_{0}^{t}\left[(\alpha-1) t(1-s)^{\alpha-2}-(t-s)^{\alpha-1}\right] y(s) d s+\int_{t}^{1}(\alpha-1) t(1-s)^{\alpha-2} y(s) d s\right. \\
& +\frac{t}{\Delta_{1}}\left[\int_{0}^{1}\left(\int_{0}^{s}(\alpha-1) \tau(1-s)^{\alpha-2} d H(\tau)\right) y(s) d s\right. \\
& \left.\left.+\int_{0}^{1}\left(\int_{s}^{1}\left[(\alpha-1) \tau(1-s)^{\alpha-2}-(\tau-s)^{\alpha-1}\right] d H(\tau)\right) y(s) d s\right]\right\} \\
= & \int_{0}^{1} g_{\alpha}(t, s) y(s) d s+\frac{t}{\Delta_{1}} \int_{0}^{1}\left(\int_{0}^{1} g_{\alpha}(\tau, s) d H(\tau)\right) y(s) d s \\
= & \int_{0}^{1} G_{\alpha}(t, s) y(s) d s,
\end{aligned}
$$

where $G_{\alpha}(t, s)$ and $g_{\alpha}(t, s)$ are defined by (2.2) and (2.3).

Now, we will prove the uniqueness of solution for BVPs (2.1). In fact, let $u_{1}(t), u_{2}(t)$ are any two solutions of (2.1). Denote $w(t)=u_{1}(t)-u_{2}(t)$, then (2.1) is changed into the following system:

$$
\left\{\begin{array}{l}
D_{0^{+}}^{\alpha} w(t)=0, \quad t \in(0,1), n-1<\alpha \leq n, n \geq 3, \\
w(0)=w^{\prime \prime}(0)=\cdots=w^{(n-1)}(0)=0, \quad w^{\prime}(1)=0 .
\end{array}\right.
$$

Similar to the above argument, we get $w(t)=0$, that is $u_{1}(t)=u_{2}(t)$, which mean that the solution for BVPs (2.1) is unique. The proof is complete.

Lemma 2.5 If $H:[0,1] \rightarrow \mathbb{R}$ is a nondecreasing function and $\Delta_{1}>0$, we also let $G_{\alpha}^{\prime}(t, s) \triangleq$ $\frac{\partial}{\partial t} G_{\alpha}(t, s), g_{\alpha}^{\prime}(t, s) \triangleq \frac{\partial}{\partial t} g_{\alpha}(t, s)$, then we have the following properties:

(1) $g_{\alpha}(t, s) \geq t^{\alpha-1} g_{\alpha}(1, s) \geq t^{\alpha-1} g_{\alpha}(t, s)$, for all $(t, s) \in[0,1] \times[0,1]$.

(2) $G_{\alpha}(t, s) \geq t^{\alpha-1} J_{\alpha}(s) \geq t^{\alpha-1} G_{\alpha}(t, s)$, for all $(t, s) \in[0,1] \times[0,1]$, where $J_{\alpha}(s)=g_{\alpha}(1, s)+$ $\frac{1}{\Delta_{1}} \int_{0}^{1} g_{\alpha}(\tau, s) d H(\tau), s \in[0,1]$.

(3) $G_{\alpha}^{\prime}(t, s) \geq 0$ for all $(t, s) \in[0,1] \times[0,1]$, and for every $\theta \in\left(0, \frac{1}{2}\right)$, we have

$$
\begin{aligned}
& \min _{t \in[\theta, 1-\theta]} g_{\alpha}^{\prime}(t, s) \geq \gamma_{1} g_{\alpha}(1, s) \geq \gamma_{2} g_{\alpha}^{\prime}\left(t^{\prime}, s\right), \quad \forall t^{\prime}, s \in[0,1], \\
& \min _{t \in[\theta, 1-\theta]} G_{\alpha}^{\prime}(t, s) \geq \gamma_{1} J_{\alpha}(s) \geq \gamma_{2} G_{\alpha}^{\prime}\left(t^{\prime}, s\right), \quad \forall t^{\prime}, s \in[0,1],
\end{aligned}
$$

where $\gamma_{1} \triangleq 1-(1-\theta)^{\alpha-2}, \gamma_{2} \triangleq \frac{\alpha-2}{\alpha-1} \gamma_{1}$

Proof (1) For $t, s \in[0,1]$, from (2.3), we have

$$
g_{\alpha}^{\prime}(t, s)= \begin{cases}\frac{(\alpha-1)(1-s)^{\alpha-2}-(\alpha-1)(t-s)^{\alpha-2}}{\Gamma(\alpha)}, & 0 \leq s \leq t \leq 1 \\ \frac{(\alpha-1)(1-s)^{\alpha-2}}{\Gamma(\alpha)}, & 0 \leq t \leq s \leq 1\end{cases}
$$

Clearly, $g_{\alpha}^{\prime}(t, s) \geq 0$ for $t, s \in[0,1]$ which indicates $g_{\alpha}(t, s)$ is increasing with respect to $t \in$ $[0,1]$. Therefore, $g_{\alpha}(t, s) \leq g_{\alpha}(1, s)$ for $t, s \in[0,1]$. 
On the other hand, for $t \geq s$, then

$$
\begin{aligned}
\frac{g_{\alpha}(t, s)}{g_{\alpha}(1, s)} & =\frac{(\alpha-1) t(1-s)^{\alpha-2}-(t-s)^{\alpha-1}}{(\alpha-1)(1-s)^{\alpha-2}-(1-s)^{\alpha-1}} \\
& \geq \frac{(\alpha-1) t^{\alpha-1}(1-s)^{\alpha-2}-(t-s)^{\alpha-1}}{(\alpha-1)(1-s)^{\alpha-2}-(1-s)^{\alpha-1}} \\
& =\frac{t^{\alpha-1}\left[(\alpha-1)(1-s)^{\alpha-2}-\left(1-\frac{s}{t}\right)^{\alpha-1}\right]}{(\alpha-1)(1-s)^{\alpha-2}-(1-s)^{\alpha-1}} \\
& \geq \frac{t^{\alpha-1}\left[(\alpha-1)(1-s)^{\alpha-2}-(1-s)^{\alpha-1}\right]}{(\alpha-1)(1-s)^{\alpha-2}-(1-s)^{\alpha-1}} \\
& =t^{\alpha-1} .
\end{aligned}
$$

Thus, for $t \leq s$, we have

$$
\frac{g_{\alpha}(t, s)}{g_{\alpha}(1, s)}=\frac{(\alpha-1) t(1-s)^{\alpha-2}}{(\alpha-1)(1-s)^{\alpha-2}-(1-s)^{\alpha-1}} \geq \frac{(\alpha-1) t(1-s)^{\alpha-2}-(t-s)^{\alpha-1}}{(\alpha-1)(1-s)^{\alpha-2}-(1-s)^{\alpha-1}} \geq t^{\alpha-1} .
$$

Therefore, $g_{\alpha}(t, s) \geq t^{\alpha-1} g_{\alpha}(1, s) \geq t^{\alpha-1} g_{\alpha}(t, s)$, for all $(t, s) \in[0,1] \times[0,1]$.

(2) From (2.2), we have

$$
\begin{aligned}
G_{\alpha}(t, s) & =g_{\alpha}(t, s)+\frac{t}{\Delta_{1}} \int_{0}^{1} g_{\alpha}(\tau, s) d H(\tau) \\
& \geq t^{\alpha-1} g_{\alpha}(1, s)+\frac{t^{\alpha-1}}{\Delta_{1}} \int_{0}^{1} g_{\alpha}(\tau, s) d H(\tau) \\
& =t^{\alpha-1} J_{\alpha}(s),
\end{aligned}
$$

where

$$
\begin{aligned}
J_{\alpha}(s) & =g_{\alpha}(1, s)+\frac{1}{\Delta_{1}} \int_{0}^{1} g_{\alpha}(\tau, s) d H(\tau) \\
& \geq g_{\alpha}(t, s)+\frac{t}{\Delta_{1}} \int_{0}^{1} g_{\alpha}(\tau, s) d H(\tau) \\
& =G_{\alpha}(t, s) .
\end{aligned}
$$

Therefore, $G_{\alpha}(t, s) \geq t^{\alpha-1} J_{\alpha}(s) \geq t^{\alpha-1} G_{\alpha}(t, s)$, for all $(t, s) \in[0,1] \times[0,1]$.

(3) From (2.4), for $t \in[0,1]$, we have $\frac{\partial^{2} g_{\alpha}(t, s)}{\partial t^{2}} \leq 0$. Thus, $g_{\alpha}^{\prime}(t, s)$ is decreasing with respect to $t \in[0,1]$. Therefore, for $\theta \in\left(0, \frac{1}{2}\right)$, we have

$$
\min _{t \in[\theta, 1-\theta]} g_{\alpha}^{\prime}(t, s) \geq g_{\alpha}^{\prime}(1-\theta, s) \geq \frac{(\alpha-1)(1-s)^{\alpha-2}-(\alpha-1)(1-\theta-s)^{\alpha-2}}{\Gamma(\alpha)} .
$$

For any $s \in[0,1]$, we get

$$
\begin{aligned}
\frac{g_{\alpha}^{\prime}(1-\theta, s)}{g_{\alpha}^{\prime}(s, s)} & \geq \frac{(\alpha-1)(1-s)^{\alpha-2}-(\alpha-1)(1-\theta-s)^{\alpha-2}}{(\alpha-1)(1-s)^{\alpha-2}} \\
& =1-\left(1-\frac{\theta}{1-s}\right)^{\alpha-2} \geq 1-(1-\theta)^{\alpha-2} \triangleq \gamma_{1} .
\end{aligned}
$$


By

$$
\frac{g_{\alpha}^{\prime}(s, s)}{g_{\alpha}(1, s)}=\frac{(\alpha-1)(1-s)^{\alpha-2}}{(\alpha-1)(1-s)^{\alpha-2}-(1-s)^{\alpha-1}}=\frac{\alpha-1}{\alpha+s-2},
$$

we derive

$$
\begin{aligned}
& \min _{t \in[\theta, 1-\theta]} g_{\alpha}^{\prime}(t, s) \geq g_{\alpha}^{\prime}(1-\theta, s) \geq \gamma_{1} g_{\alpha}^{\prime}(s, s)=\frac{\alpha-1}{\alpha+s-2} \gamma_{1} g_{\alpha}(1, s) \geq \gamma_{1} g_{\alpha}(1, s), \\
& g_{\alpha}^{\prime}(t, s) \leq g_{\alpha}^{\prime}(s, s)=\frac{(\alpha-1)(1-s)^{\alpha-2}}{\Gamma(\alpha)}=\frac{\alpha-1}{\alpha+s-2} g_{\alpha}(1, s) \leq \frac{\alpha-1}{\alpha-2} g_{\alpha}(1, s) .
\end{aligned}
$$

Therefore,

$$
\min _{t \in[\theta, 1-\theta]} g_{\alpha}^{\prime}(t, s) \geq \gamma_{1} g_{\alpha}(1, s) \geq \frac{\alpha-2}{\alpha-1} \gamma_{1} g_{\alpha}^{\prime}\left(t^{\prime}, s\right) \triangleq \gamma_{2} g_{\alpha}^{\prime}\left(t^{\prime}, s\right), \quad \forall t^{\prime}, s \in[0,1]
$$

From (2.2), for $\forall t, s \in[0,1]$, we have

$$
\begin{aligned}
G_{\alpha}^{\prime}(t, s) & =g_{\alpha}^{\prime}(t, s)+\frac{1}{\Delta_{1}} \int_{0}^{1} g_{\alpha}(\tau, s) d H(\tau) \\
& \leq \frac{\alpha-1}{\alpha-2} g_{\alpha}(1, s)+\frac{1}{\Delta_{1}} \int_{0}^{1} g_{\alpha}(\tau, s) d H(\tau) \\
& \leq \frac{\alpha-1}{\alpha-2}\left[g_{\alpha}(1, s)+\frac{1}{\Delta_{1}} \int_{0}^{1} g_{\alpha}(\tau, s) d H(\tau)\right] \\
& =\frac{\alpha-1}{\alpha-2} J_{\alpha}(s) .
\end{aligned}
$$

According to $g_{\alpha}^{\prime}(t, s) \geq 0, \Delta_{1}>0$, it is clearly that $G_{\alpha}^{\prime}(t, s) \geq 0$. For $\forall t^{\prime}, s \in[0,1]$, we obtain

$$
\begin{aligned}
\min _{t \in[\theta, 1-\theta]} G_{\alpha}^{\prime}(t, s) & =\min _{t \in[\theta, 1-\theta]} g_{\alpha}^{\prime}(t, s)+\frac{1}{\Delta_{1}} \int_{0}^{1} g_{\alpha}(\tau, s) d H(\tau) \\
& \geq \gamma_{1} g_{\alpha}(1, s)+\frac{1}{\Delta_{1}} \int_{0}^{1} g_{\alpha}(\tau, s) d H(\tau) \\
& \geq \gamma_{1}\left[g_{\alpha}(1, s)+\frac{1}{\Delta_{1}} \int_{0}^{1} g_{\alpha}(\tau, s) d H(\tau)\right] \\
& =\gamma_{1} J_{\alpha}(s) \geq \frac{\alpha-2}{\alpha-1} \gamma_{1} G_{\alpha}^{\prime}\left(t^{\prime}, s\right)=\gamma_{2} G_{\alpha}^{\prime}\left(t^{\prime}, s\right) .
\end{aligned}
$$

The proof of Lemma 2.5 is complete.

From Lemma 2.5, we have the following lemma.

Lemma 2.6 If $H:[0,1] \rightarrow \mathbb{R}$ is a nondecreasing function and $\Delta_{1}>0$, then the Green's functions $G_{\alpha}, G_{\alpha}^{\prime}$ of BVPs $(2.1)$ are continuous on $[0,1] \times[0,1]$ and satisfy $G_{\alpha}(t, s), G_{\alpha}^{\prime}(t, s) \geq 0$ for all $(t, s) \in[0,1] \times[0,1]$. Moreover, if $y \in C([0,1])$ satisfies $y(t) \geq 0$ for all $t \in[0,1]$, then the unique solution $u(t)$ of BVPs (2.1) satisfies $u(t) \geq 0, \min _{t \in[\theta, 1-\theta]} u(t) \geq \theta^{\alpha-1} \max _{t^{\prime} \in[0,1]} u\left(t^{\prime}\right)$, $u^{\prime}(t)=\int_{0}^{1} G_{\alpha}^{\prime}(t, s) y(s) d s \geq 0$ for all $t \in[0,1]$ and $\min _{t \in[\theta, 1-\theta]} u^{\prime}(t) \geq \gamma_{2} \max _{t^{\prime} \in[0,1]} u^{\prime}\left(t^{\prime}\right)$. 
We can also formulate similar results as Lemmas 2.4-2.6 above for the fractional differential equation

$$
\left\{\begin{array}{l}
D_{0^{+}}^{\beta} v(t)+h(t)=0, \quad t \in(0,1), m-1<\beta \leq m, m \geq 3, \\
v(0)=v^{\prime \prime}(0)=\cdots=v^{(m-1)}(0)=0, \quad v^{\prime}(1)=\int_{0}^{1} v(s) d K(s),
\end{array}\right.
$$

where $m \in \mathbb{N}, m \geq 3, K:[0,1] \rightarrow \mathbb{R}$ is a nondecreasing function and $h \in C([0,1])$. In a similar manner as $\Delta_{1}, \gamma_{1}, \gamma_{2}, g_{\alpha}, g_{\alpha}^{\prime}, G_{\alpha}, G_{\alpha}^{\prime}$ and $J_{\alpha}$, we introduce $\Delta_{2}, \gamma_{1}^{\prime}, \gamma_{2}^{\prime}, g_{\beta}, g_{\beta}^{\prime}, G_{\beta}$, $G_{\beta}^{\prime}$ and $J_{\beta}$ the corresponding constants and functions for BVPs (2.5) defined by $\Delta_{2} \triangleq$ $1-\int_{0}^{1} s d K(s) \neq 0, \gamma_{1}^{\prime} \triangleq 1-(1-\theta)^{\beta-2}, \gamma_{2}^{\prime} \triangleq \frac{\beta-2}{\beta-1} \gamma_{1}^{\prime}, G_{\beta}(t, s)=g_{\beta}(t, s)+\frac{t}{\Delta_{2}} \int_{0}^{1} g_{\beta}(\tau, s) d K(\tau)$, $G_{\beta}^{\prime}(t, s) \triangleq \frac{\partial}{\partial t} G_{\beta}(t, s), g_{\beta}^{\prime}(t, s) \triangleq \frac{\partial}{\partial t} g_{\beta}(t, s), J_{\beta}(s)=g_{\beta}(1, s)+\frac{1}{\Delta_{2}} \int_{0}^{1} g_{\beta}(\tau, s) d K(\tau)$,

$$
g_{\beta}(t, s)= \begin{cases}\frac{(\beta-1) t(1-s)^{\beta-2}-(t-s)^{\beta-1}}{\Gamma(\beta)}, & 0 \leq s \leq t \leq 1 \\ \frac{(\beta-1) t(1-)^{\beta-2}}{\Gamma(\beta)}, & 0 \leq t \leq s \leq 1\end{cases}
$$

\section{Existence and nonexistence of positive solutions}

In this section, we will discuss the existence and nonexistence of positive solutions to the BVPs (1.1)-(1.2) under various assumptions on $f$ and $g$.

We present the assumptions that we shall use in the sequel.

$\left(\mathrm{H}_{1}\right) H, K:[0,1] \rightarrow \mathbb{R}$ are nondecreasing functions, $\Delta_{1}=1-\int_{0}^{1} s d H(s)>0, \Delta_{2}=1-$ $\int_{0}^{1} s d K(s)>0$.

$\left(\mathrm{H}_{2}\right)$ The functions $f:[0,1] \times[0, \infty)^{4} \rightarrow[0, \infty), g:[0,1] \times[0, \infty)^{2} \rightarrow[0, \infty)$ are continuous and $f(t, 0,0,0,0)=g(t, 0,0)=0$ for all $t \in[0,1]$.

For simplicity, we introduce some important notations as follows:

$$
\begin{aligned}
& f^{0}=\limsup _{x+y+z+w \rightarrow 0^{+}} \max _{t \in[0,1]} \frac{f(t, x, y, z, w)}{x+y+z+w}, \quad g^{0}=\limsup _{x+y \rightarrow 0^{+}} \max _{t \in[0,1]} \frac{g(t, x, y)}{x+y}, \\
& f_{0}=\liminf _{x+y+z+w \rightarrow 0^{+}} \min _{t \in[\theta, 1-\theta]} \frac{f(t, x, y, z, w)}{x+y+z+w}, \quad g_{0}=\liminf _{x+y \rightarrow 0^{+}} \min _{t \in[\theta, 1-\theta]} \frac{g(t, x, y)}{x+y}, \\
& f^{\infty}=\limsup _{x+y+z+w \rightarrow+\infty} \max _{t \in[0,1]} \frac{f(t, x, y, z, w)}{x+y+z+w}, \quad g^{\infty}=\limsup _{x+y \rightarrow+\infty} \max _{t \in[0,1]} \frac{g(t, x, y)}{x+y}, \\
& f_{\infty}=\liminf _{x+y+z+w \rightarrow+\infty} \min _{t \in[\theta, 1-\theta]} \frac{f(t, x, y, z, w)}{x+y+z+w}, \quad g_{\infty}=\liminf _{x+y \rightarrow+\infty} \min _{t \in[\theta, 1-\theta]} \frac{g(t, x, y)}{x+y}, \\
& A_{1}=\frac{2 \alpha-3}{\alpha-2} \int_{0}^{1} J_{\alpha}(s) d s, \quad A_{2}=\left(\theta^{\alpha-1}+\gamma_{1}\right) \int_{\theta}^{1-\theta} J_{\alpha}(s) d s, \\
& B_{1}=\frac{2 \beta-3}{\beta-2} \int_{0}^{1} J_{\beta}(s) d s, \quad B_{2}=\gamma_{0}\left(\theta^{\beta-1}+\gamma_{1}^{\prime}\right) \int_{\theta}^{1-\theta} J_{\beta}(s) d s,
\end{aligned}
$$

where $\gamma_{0}=\min \left\{\theta^{\alpha-1}, \gamma_{2}\right\}$.

Let $E=C^{1}[0,1]$ be endowed with the norm

$$
\|u\|=\max _{t \in[0,1]}|u(t)|+\max _{t \in[0,1]}\left|u^{\prime}(t)\right|=\|u\|_{0}+\left\|u^{\prime}\right\|_{0} .
$$


Let the cone $P \subset E$ and the operators $T: P \rightarrow P$ be, respectively, defined by

$$
P=\left\{u \in E: u(t), u^{\prime}(t) \geq 0, \min _{t \in[\theta, 1-\theta]} u(t) \geq \theta^{\alpha-1}\|u\|_{0}, \min _{t \in[\theta, 1-\theta]} u^{\prime}(t) \geq \gamma_{2}\left\|u^{\prime}\right\|_{0}\right\}
$$

and

$$
\begin{aligned}
(T u)(t)= & \int_{0}^{1} G_{\alpha}(t, s) f\left(s, u(s), \int_{0}^{1} G_{\beta}(s, \tau) g\left(\tau, u(\tau), u^{\prime}(\tau)\right) d \tau, u^{\prime}(s),\right. \\
& \left.\int_{0}^{1} G_{\beta}^{\prime}(s, \tau) g\left(\tau, u(\tau), u^{\prime}(\tau)\right) d \tau\right) d s, \quad t \in[0,1] .
\end{aligned}
$$

It is easy to see that if $x(t)$ is a fixed point of $T$, then BVPs (1.1)-(1.2) have a pair of solution $(u, v)$ expressed as

$$
\begin{cases}u(t)=x(t), & t \in[0,1] \\ v(t)=\int_{0}^{1} G_{\beta}(t, s) g\left(s, x(s), x^{\prime}(s)\right) d s, & t \in[0,1] .\end{cases}
$$

Theorem 3.1 Assume that $\left(\mathrm{H}_{1}\right)-\left(\mathrm{H}_{2}\right)$ hold. Assume $A_{1} f^{0}<\frac{1}{2}<A_{2} f_{\infty}$, and $B_{1} g^{0}<1<B_{2} g_{\infty}$. Then BVPs (1.1)-(1.2) have at least a pair of positive solutions $(u(t), v(t))$.

Proof In view of $A_{1} f^{0}<\frac{1}{2}$ and $B_{1} g^{0}<1$, there exists $\varepsilon_{1}>0$ such that

$$
A_{1}\left(f^{0}+\varepsilon_{1}\right) \leq \frac{1}{2}, \quad B_{1}\left(g^{0}+\varepsilon_{1}\right) \leq 1 .
$$

By the definition of $f^{0}, g^{0}$, we may choose $\sigma_{1}>0$ such that, for $t \in[0,1], 0 \leq x+y \leq x+y+$ $z+w \leq \sigma_{1}$, we have

$$
f(t, x, y, z, w) \leq\left(f^{0}+\varepsilon_{1}\right)(x+y+z+w), \quad g(t, x, y) \leq\left(g^{0}+\varepsilon_{1}\right)(x+y) .
$$

Let $\Omega_{1}=\left\{u \in P:\|u\|<\sigma_{1}\right\}$. Define the operator $T: \Omega_{1} \rightarrow \Omega_{1}$ the same as (3.3). We shall prove the theorem through two steps.

Step 1. We assert that $T: \Omega_{1} \rightarrow \Omega_{1}$ is completely continuous. In fact, by the definition $T$, it is easy to see that $T$ is continuous in $\Omega_{1}$. It follows from (3.4), (3.5), and Lemma 2.5 that, for any $u \in \Omega_{1}, s \in[0,1]$,

$$
\begin{aligned}
& \int_{0}^{1}\left(G_{\beta}(s, \tau)+G_{\beta}^{\prime}(s, \tau)\right) g\left(\tau, u(\tau), u^{\prime}(\tau)\right) d \tau \\
& \quad \leq \int_{0}^{1}\left(J_{\beta}(\tau)+\frac{\beta-1}{\beta-2} J_{\beta}(\tau)\right)\left(g^{0}+\varepsilon_{1}\right)\left(u(\tau)+u^{\prime}(\tau)\right) d \tau \\
& \quad \leq \frac{(2 \beta-3)\left(g^{0}+\varepsilon_{1}\right)\|u\|}{\beta-2} \int_{0}^{1} J_{\beta}(\tau) d \tau<\sigma_{1},
\end{aligned}
$$

which implies that

$$
\begin{aligned}
\|T u\| & =\max _{0 \leq t \leq 1}|T u(t)|+\max _{0 \leq t \leq 1}\left|(T u)^{\prime}(t)\right| \\
& =\int_{0}^{1}\left(G_{\alpha}(t, s)+G_{\alpha}^{\prime}(t, s)\right) f\left(s, u(s), \int_{0}^{1} G_{\beta}(s, \tau) g\left(\tau, u(\tau), u^{\prime}(\tau)\right) d \tau, u^{\prime}(s),\right.
\end{aligned}
$$




$$
\begin{aligned}
& \left.\int_{0}^{1} G_{\beta}^{\prime}(s, \tau) g\left(\tau, u(\tau), u^{\prime}(\tau)\right) d \tau\right) d s \\
\leq & \int_{0}^{1}\left(J_{\alpha}(s)+\frac{\alpha-1}{\alpha-2} J_{\alpha}(s)\right)\left(f^{0}+\varepsilon_{1}\right)\left(\int_{0}^{1} G_{\beta}(s, \tau) g\left(\tau, u(\tau), u^{\prime}(\tau)\right) d \tau\right. \\
& \left.+\int_{0}^{1} G_{\beta}^{\prime}(s, \tau) g\left(\tau, u(\tau), u^{\prime}(\tau)\right) d \tau+u(s)+u^{\prime}(s)\right) d s \\
\leq & \frac{(2 \alpha-3)\left(f^{0}+\varepsilon_{1}\right)}{\alpha-2} \int_{0}^{1} J_{\alpha}(s)\left(\frac{(2 \beta-3)\left(g^{0}+\varepsilon_{1}\right)}{\beta-2}\right. \\
& \left.\times \int_{0}^{1} J_{\beta}(\tau)\left(u(\tau)+u^{\prime}(\tau)\right) d \tau+\|u\|\right) d s \\
\leq & \frac{2 \alpha-3}{\alpha-2} \int_{0}^{1} J_{\alpha}(s) d s\left(f^{0}+\varepsilon_{1}\right)\left[\frac{2 \beta-3}{\beta-2} \int_{0}^{1} J_{\beta}(s) d s\left(g^{0}+\varepsilon_{1}\right)+1\right]\|u\| \\
= & A_{1}\left(f^{0}+\varepsilon_{1}\right)\left[B_{1}\left(g^{0}+\varepsilon_{1}\right)+1\right]\|u\| \leq\|u\|<\sigma_{1} .
\end{aligned}
$$

Thus, we show that $T\left(\Omega_{1}\right) \subset \Omega_{1}$ and $T\left(\Omega_{1}\right)$ is uniformly bounded.

Next, we prove that $T: \Omega_{1} \rightarrow \Omega_{1}$ is equicontinuous in [0,1], that is, for any $u \in \Omega_{1}$, $t_{1}, t_{2} \in[0,1], \forall \epsilon>0, \exists \delta=\delta(\epsilon)>0$, when $\left|t_{1}-t_{2}\right|<\delta$, then $\left|(T u)\left(t_{1}\right)-(T u)\left(t_{2}\right)\right|<\epsilon$. Indeed, take $\delta=\delta(\epsilon)=\frac{(2 \alpha-3) \epsilon}{(\alpha-1) \sigma_{1}}$, we have

$$
\begin{aligned}
\left|(T u)\left(t_{1}\right)-(T u)\left(t_{2}\right)\right| & \mid \int_{0}^{1}\left(G_{\alpha}\left(t_{1}, s\right)-G_{\alpha}\left(t_{2}, s\right)\right) f\left(s, u(s), \int_{0}^{1} G_{\beta}(s, \tau) g\left(\tau, u(\tau), u^{\prime}(\tau)\right) d \tau, u^{\prime}(s),\right. \\
& \left.\int_{0}^{1} G_{\beta}^{\prime}(s, \tau) g\left(\tau, u(\tau), u^{\prime}(\tau)\right) d \tau\right) d s \mid \\
\leq & \int_{0}^{1}\left|G_{\alpha}^{\prime}(\xi, s)\right|\left|t_{1}-t_{2}\right| f\left(s, u(s), \int_{0}^{1} G_{\beta}(s, \tau) g\left(\tau, u(\tau), u^{\prime}(\tau)\right) d \tau, u^{\prime}(s),\right. \\
& \left.\int_{0}^{1} G_{\beta}^{\prime}(s, \tau) g\left(\tau, u(\tau), u^{\prime}(\tau)\right) d \tau\right) d s \mid \\
= & {\left[\int _ { 0 } ^ { 1 } G _ { \alpha } ^ { \prime } ( \xi , s ) f \left(s, u(s), \int_{0}^{1} G_{\beta}(s, \tau) g\left(\tau, u(\tau), u^{\prime}(\tau)\right) d \tau, u^{\prime}(s),\right.\right.} \\
& \left.\left.\int_{0}^{1} G_{\beta}^{\prime}(s, \tau) g\left(\tau, u(\tau), u^{\prime}(\tau)\right) d \tau\right) d s\right]\left|t_{1}-t_{2}\right| \\
\leq & {\left[\frac { ( \alpha - 1 ) ( f ^ { 0 } + \varepsilon _ { 1 } ) } { \alpha - 2 } \int _ { 0 } ^ { 1 } J _ { \alpha } ( s ) \left(\int_{0}^{1} G_{\beta}(s, \tau) g\left(\tau, u(\tau), u^{\prime}(\tau)\right) d \tau\right.\right.} \\
& \left.\left.+\int_{0}^{1} G_{\beta}^{\prime}(s, \tau) g\left(\tau, u(\tau), u^{\prime}(\tau)\right) d \tau+u(s)+u^{\prime}(s)\right) d s\right]\left|t_{1}-t_{2}\right| \\
\leq & \frac{2 \alpha-3}{\alpha-2} \int_{0}^{1} J_{\alpha}(s) d s\left(f^{0}+\varepsilon_{1}\right) \\
& \times\left[\frac{2 \beta-3}{\beta-2} \int_{0}^{1} J_{\beta}(s) d s\left(g^{0}+\varepsilon_{1}\right)+1\right]\|u\| \frac{\alpha-1}{2 \alpha-3}\left|t_{1}-t_{2}\right| \\
= & A_{1}\left(f^{0}+\varepsilon_{1}\right)\left[B_{1}\left(g^{0}+\varepsilon_{1}\right)+1\right]\|u\| \frac{\alpha-1}{2 \alpha-3}\left|t_{1}-t_{2}\right|<\frac{(\alpha-1) \sigma_{1}}{2 \alpha-3}\left|t_{1}-t_{2}\right|<\epsilon .
\end{aligned}
$$


Step 2. Now we verify condition (i) or (ii) of Lemma 2.2. In fact, for all $u \in P \cap \partial \Omega_{1}$, $s \in[0,1]$, similar to the argument of (3.6), we get

$$
\|T u\| \leq\|u\|, \quad u \in P \cap \partial \Omega_{1} .
$$

On the other hand, since $\frac{1}{2}<A_{2} f_{\infty}$ and $1<B_{2} g_{\infty}$, there exists $\varepsilon_{2}>0$ such that

$$
A_{2}\left(f_{\infty}-\varepsilon_{2}\right) \geq \frac{1}{2}, \quad B_{2}\left(g_{\infty}-\varepsilon_{2}\right) \geq 1
$$

By the definition of $f_{\infty}, g_{\infty}$, we can choose $\sigma_{2}^{\prime}>\sigma_{1}$ such that, for $t \in[\theta, 1-\theta], \sigma_{2}^{\prime} \leq x+y \leq$ $x+y+z+w<\infty$, we have

$$
f(t, x, y, z, w) \geq\left(f_{\infty}-\varepsilon_{2}\right)(x+y), \quad g(t, x, y) \geq\left(g_{\infty}-\varepsilon_{2}\right)(x+y) .
$$

Let $\sigma_{2}=\max \left\{\sigma_{1}, \frac{\sigma_{2}^{\prime}}{\gamma_{0}}\right\}=\frac{\sigma_{2}^{\prime}}{\gamma_{0}}$, where $\gamma_{0}=\min \left\{\theta^{\alpha-1}, \gamma_{2}\right\}$. Set $\Omega_{2}=\left\{u \in P:\|u\|<\sigma_{2}\right\}$. Define the operator $T: \Omega_{2} \rightarrow \Omega_{2}$ as (3.3). Similar to the above discussion of $T: \Omega_{1} \rightarrow \Omega_{1}$, we know that $T: \Omega_{2} \rightarrow \Omega_{2}$ is completely continuous. Then for $t \in[\theta, 1-\theta], u \in P \cap \partial \Omega_{2}$ implies that

$$
u(t)+u^{\prime}(t) \geq \theta^{\alpha-1}\|u\|_{0}+\gamma_{2}\left\|u^{\prime}\right\|_{0} \geq \min \left\{\theta^{\alpha-1}, \gamma_{2}\right\}\left(\|u\|_{0}+\left\|u^{\prime}\right\|_{0}\right)=\gamma_{0}\|u\| \geq \sigma_{2}^{\prime}
$$

It follows from (3.8), (3.9), and Lemma 2.5 that, for any $u \in P \cap \partial \Omega_{2}, s \in[\theta, 1-\theta]$, we have

$$
\begin{aligned}
& \int_{0}^{1}\left(G_{\beta}(s, \tau)+G_{\beta}^{\prime}(s, \tau)\right) g\left(\tau, u(\tau), u^{\prime}(\tau)\right) d \tau \\
& \quad \geq \int_{\theta}^{1-\theta}\left(\theta^{\beta-1} J_{\beta}(\tau)+\gamma_{1}^{\prime} J_{\beta}(\tau)\right)\left(g_{\infty}-\varepsilon_{2}\right)\left(u(\tau)+u^{\prime}(\tau)\right) d \tau \\
& \quad \geq\left(g_{\infty}-\varepsilon_{2}\right) \gamma_{0}\|u\|\left(\theta^{\beta-1}+\gamma_{1}^{\prime}\right) \int_{\theta}^{1-\theta} J_{\beta}(\tau) d \tau \\
& \quad=B_{2}\left(g_{\infty}-\varepsilon_{2}\right)\|u\| \geq\|u\| .
\end{aligned}
$$

Then, for $t \in[\theta, 1-\theta]$, by (3.8)-(3.10) and Lemma 2.5, we get

$$
\begin{aligned}
\|T u\|= & \max _{0 \leq t \leq 1}|T u(t)|+\max _{0 \leq t \leq 1}\left|(T u)^{\prime}(t)\right| \\
= & \int_{0}^{1}\left(G_{\alpha}(t, s)+G_{\alpha}^{\prime}(t, s)\right) f\left(s, u(s), \int_{0}^{1} G_{\beta}(s, \tau) g\left(\tau, u(\tau), u^{\prime}(\tau)\right) d \tau, u^{\prime}(s),\right. \\
& \left.\int_{0}^{1} G_{\beta}^{\prime}(s, \tau) g\left(\tau, u(\tau), u^{\prime}(\tau)\right) d \tau\right) d s \\
\geq & \int_{\theta}^{1-\theta}\left(\theta^{\alpha-1} J_{\alpha}(s)+\gamma_{1} J_{\alpha}(s)\right)\left(f_{\infty}-\varepsilon_{2}\right)\left(\int_{0}^{1} G_{\beta}(s, \tau) g\left(\tau, u(\tau), u^{\prime}(\tau)\right) d \tau\right. \\
& \left.+\int_{0}^{1} G_{\beta}^{\prime}(s, \tau) g\left(\tau, u(\tau), u^{\prime}(\tau)\right) d \tau+u(s)+u^{\prime}(s)\right) d s \\
\geq & A_{2}\left(f_{\infty}-\varepsilon_{2}\right)\left[B_{2}\left(g_{\infty}-\varepsilon_{2}\right)+1\right]\|u\| \geq\|u\|
\end{aligned}
$$


which implies that

$$
\|T u\| \geq\|u\|, \quad u \in P \cap \partial \Omega_{2} .
$$

By (3.7), (3.11), and condition (i) of Lemma 2.2, we know that $T$ has at least one fixed point $u_{1} \in P \cap\left(\bar{\Omega}_{2} \backslash \Omega_{1}\right)$. Consequently, BVPs (1.1)-(1.2) have at least a pair of positive solution $(u, v) \in P \times P$, here $u(t)=u_{1}(t), v(t)=\int_{0}^{1} G_{\beta}(t, s) g\left(s, u_{1}(s), u_{1}^{\prime}(s)\right) d s$. The proof is complete.

Similarly, we can get the following theorem.

Theorem 3.2 Assume that $\left(\mathrm{H}_{1}\right)-\left(\mathrm{H}_{2}\right)$ hold. Assume $A_{1} f^{\infty}<\frac{1}{2}<A_{2} f_{0}$ and $B_{1} g^{\infty}<1<B_{2} g_{0}$. Then BVPs (1.1)-(1.2) have at least a pair of positive solution.

Theorem 3.3 Assume that $\left(\mathrm{H}_{1}\right)-\left(\mathrm{H}_{2}\right)$ hold. If $A_{2} f_{0}>\frac{1}{2}, B_{2} g_{0}>1, A_{2} f_{\infty}>\frac{1}{2}, B_{2} g_{\infty}>1$, $B_{2} g^{0}<2$, and there exists a constant $\mu>0$ such that

$$
\begin{aligned}
& \max \{g(t, x, y): t \in[0,1], x+y \in[0, \mu]\}<\frac{\mu}{B_{1}} \\
& \max \{f(t, x, y, z, w): t \in[0,1], x+y+z+w \in[0, \mu]\}<\frac{\mu}{A_{1}} .
\end{aligned}
$$

Then BVPs (1.1)-(1.2) have at least two pairs of positive solutions.

Proof In view of $A_{2} f_{0}>1$ and $B_{2} g_{0}>1$, there exists $\varepsilon>0$ such that

$$
A_{2}\left(f_{0}-\varepsilon\right) \geq \frac{1}{2}, \quad B_{2}\left(g_{0}-\varepsilon\right) \geq 1 .
$$

By the definition of $f_{0}, g_{0}$, we may choose $\hat{\sigma}_{1}>0$ such that, for $t \in[\theta, 1-\theta], 0 \leq x+y \leq$ $x+y+z+w \leq \hat{\sigma}_{1}$, we have

$$
f(t, x, y, z, w) \geq\left(f_{0}-\varepsilon\right)(x+y+z+w), \quad g(t, x, y) \geq\left(g_{0}-\varepsilon\right)(x+y) .
$$

Moreover, from $B_{2} g^{0}<2$, take $\rho_{1}$ satisfying $0<\rho_{1}<\frac{B_{2}}{2 B_{1}} \hat{\sigma}_{1}<\mu$ such that

$$
g(t, x, y) \leq g^{0} \times(x+y) \leq \frac{2 \rho_{1}}{B_{2}}, \quad \forall t \in[0,1], x+y \in\left[0, \rho_{1}\right]
$$

Set $\Omega_{1}=\left\{u \in P:\|u\|<\rho_{1}\right\}$. Define the operator $T: \Omega_{1} \rightarrow \Omega_{1}$ as (3.3). Similar to the discussion of Theorem 3.1, we know that $T: \Omega_{1} \rightarrow \Omega_{1}$ is completely continuous. It follows from (3.14)-(3.15) and Lemma 2.5 that, for any $u \in P \cap \partial \Omega_{1}, s \in[0,1]$,

$$
\begin{aligned}
& \int_{0}^{1}\left(G_{\beta}(s, \tau)+G_{\beta}^{\prime}(s, \tau)\right) g\left(\tau, u(\tau), u^{\prime}(\tau)\right) d \tau \\
& \quad \leq \frac{2 \rho_{1}}{B_{2}} \int_{0}^{1}\left(J_{\beta}(\tau)+\frac{\beta-1}{\beta-2} J_{\beta}(\tau)\right) d \tau=\frac{2 B_{1}}{B_{2}} \rho_{1}<\hat{\sigma}_{1} .
\end{aligned}
$$


Then, for $t \in[\theta, 1-\theta]$, by (3.14)-(3.16) and Lemma 2.5, we obtain

$$
\begin{aligned}
& \|T u\|=\max _{0 \leq t \leq 1}|T u(t)|+\max _{0 \leq t \leq 1}\left|(T u)^{\prime}(t)\right| \\
& =\int_{0}^{1}\left(G_{\alpha}(t, s)+G_{\alpha}^{\prime}(t, s)\right) f\left(s, u(s), \int_{0}^{1} G_{\beta}(s, \tau) g\left(\tau, u(\tau), u^{\prime}(\tau)\right) d \tau, u^{\prime}(s),\right. \\
& \left.\int_{0}^{1} G_{\beta}^{\prime}(s, \tau) g\left(\tau, u(\tau), u^{\prime}(\tau)\right) d \tau\right) d s \\
& \geq \int_{\theta}^{1-\theta}\left(\theta^{\alpha-1} J_{\alpha}(s)+\gamma_{1} J_{\alpha}(s)\right)\left(f_{0}-\varepsilon\right)\left(\int_{0}^{1} G_{\beta}(s, \tau) g\left(\tau, u(\tau), u^{\prime}(\tau)\right) d \tau\right. \\
& \left.+\int_{0}^{1} G_{\beta}^{\prime}(s, \tau) g\left(\tau, u(\tau), u^{\prime}(\tau)\right) d \tau+u(s)+u^{\prime}(s)\right) d s \\
& \geq\left(\theta^{\alpha-1}+\gamma_{1}\right) \int_{\theta}^{1-\theta} J_{\alpha}(s)\left(f_{0}-\varepsilon\right) \\
& \times\left(\int_{\theta}^{1-\theta}\left(G_{\beta}(s, \tau)+G_{\beta}^{\prime}(s, \tau)\right) g\left(\tau, u(\tau), u^{\prime}(\tau)\right) d \tau+u(s)+u^{\prime}(s)\right) d s \\
& \geq\left(\theta^{\alpha-1}+\gamma_{1}\right) \int_{\theta}^{1-\theta} J_{\alpha}(s)\left(f_{0}-\varepsilon\right) \\
& \times\left(\left(\theta^{\beta-1}+\gamma_{1}^{\prime}\right) \int_{\theta}^{1-\theta} J_{\beta}(\tau)\left(g_{0}-\varepsilon\right)\left(u(\tau)+u^{\prime}(\tau)\right) d \tau+u(s)+u^{\prime}(s)\right) d s \\
& \geq\left(\theta^{\alpha-1}+\gamma_{1}\right) \int_{\theta}^{1-\theta} J_{\alpha}(s) d s\left(f_{0}-\varepsilon\right) \\
& \times\left[\left(\theta^{\beta-1}+\gamma_{1}^{\prime}\right) \int_{\theta}^{1-\theta} J_{\beta}(s) d s\left(g_{0}-\varepsilon\right) \gamma_{0}+1\right]\|u\| \\
& =A_{2}\left(f_{0}-\varepsilon\right)\left[B_{2}\left(g_{0}-\varepsilon\right)+1\right]\|u\| \geq\|u\| \text {. }
\end{aligned}
$$

Therefore,

$$
\|T u\| \geq\|u\|, \quad u \in P \cap \partial \Omega_{1} .
$$

Secondly, according to $A_{2} f_{\infty}>\frac{1}{2}$ and $B_{2} g_{\infty}>1$, similar to the proof of (3.11), choosing $\sigma_{2}>\mu$, setting $\Omega_{2}=\left\{u \in P:\|u\|<\sigma_{2}\right\}$ and defining the operator $T: \Omega_{2} \rightarrow \Omega_{2}$ as (3.3), we easily get

$$
\|T u\| \geq\|u\|, \quad u \in P \cap \partial \Omega_{2} .
$$

On the other hand, let $\Omega_{3}=\{u \in P:\|u\|<\mu\}$. Define the operator $T: \Omega_{3} \rightarrow \Omega_{3}$ as (3.3). Similar to the discussion of Theorem 3.1, we know that $T: \Omega_{3} \rightarrow \Omega_{3}$ is completely continuous. Then, for any $u \in P \cap \partial \Omega_{3}$, it follows from (3.12) and (3.13) that

$$
\int_{0}^{1}\left(G_{\beta}(s, \tau)+G_{\beta}^{\prime}(s, \tau)\right) g\left(\tau, u(\tau), u^{\prime}(\tau)\right) d \tau<\frac{\mu}{B_{1}} \int_{0}^{1}\left(J_{\beta}(\tau)+\frac{\beta-1}{\beta-2} J_{\beta}(\tau)\right) d \tau=\mu
$$


and

$$
\begin{aligned}
\|T u\|= & \max _{0 \leq t \leq 1}|T u(t)|+\max _{0 \leq t \leq 1}\left|(T u)^{\prime}(t)\right| \\
= & \int_{0}^{1}\left(G_{\alpha}(t, s)+G_{\alpha}^{\prime}(t, s)\right) f\left(s, u(s), \int_{0}^{1} G_{\beta}(s, \tau) g\left(\tau, u(\tau), u^{\prime}(\tau)\right) d \tau, u^{\prime}(s),\right. \\
& \left.\int_{0}^{1} G_{\beta}^{\prime}(s, \tau) g\left(\tau, u(\tau), u^{\prime}(\tau)\right) d \tau\right) d s \\
< & \int_{0}^{1}\left(J_{\alpha}(s)+\frac{\alpha-1}{\alpha-2} J_{\alpha}(s)\right) d s \frac{\mu}{A_{1}}=\mu=\|u\| .
\end{aligned}
$$

So

$\|T u\|<\|u\|, \quad u \in P \cap \partial \Omega_{3}$.

By (3.17), (3.19), and condition (ii) of Lemma 2.2, we know that $T$ has at least a fixed point in $u_{1} \in P \cap\left(\bar{\Omega}_{3} \backslash \Omega_{1}\right)$, that is, $\rho_{1} \leq\left\|u_{1}\right\| \leq \mu$. Equations (3.18) and (3.19) together with condition (i) of Lemma 2.2 imply that $T$ has at least one fixed point $u_{2} \in P \cap\left(\bar{\Omega}_{2} \backslash \Omega_{3}\right)$, namely, $\mu \leq\left\|u_{2}\right\| \leq \sigma_{2}$. It is worth noting that $\rho_{1}<\mu<\sigma_{2}$, and (3.19) is a strict inequality, that is to say, the operator $T$ has not the fixed point on the boundary $\partial \Omega_{3}$. So we conclude that BVPs (1.1)-(1.2) have at least two pairs of positive solutions $\left(u_{1}, v_{1}\right)$ and $\left(u_{2}, v_{2}\right)$ with the properties of $\rho_{1} \leq\left\|u_{1}\right\|<\mu<\left\|u_{2}\right\| \leq \sigma_{2}$ and $v_{i}(t)=\int_{0}^{1} G_{\beta}(t, s) g\left(s, u_{i}(s), u_{i}^{\prime}(s)\right) d s(i=1,2)$. The proof is complete.

Similarly, we get the following theorem.

Theorem 3.4 Assume that $\left(\mathrm{H}_{1}\right)-\left(\mathrm{H}_{2}\right)$ hold. Assume $A_{1} f^{0}<\frac{1}{2}, B_{1} g^{0}<1, A_{1} f^{\infty}<\frac{1}{2}, B_{2} g_{\infty}>$ $\gamma_{0}$, and there is a $\eta>0$ such that

$$
\begin{aligned}
& \min \left\{g(t, x, y): t \in[\theta, 1-\theta], x+y \in\left[\gamma_{0} \eta, \infty\right)\right\}>\frac{\gamma_{0}^{2} \eta}{B_{2}} ; \\
& \min \left\{f(t, x, y, z, w): t \in[\theta, 1-\theta], x+y+z+w \in\left[\gamma_{0} \eta, \infty\right)\right\}>\frac{\eta}{A_{2}} .
\end{aligned}
$$

Then BVPs (1.1)-(1.2) have at least two pairs of positive solutions.

Theorem 3.5 Assume that $\left(\mathrm{H}_{1}\right)-\left(\mathrm{H}_{2}\right)$ hold. Further suppose that $f(t, x, y, z$, w) and $g(t, x, y)$ are nondecreasing functions with respect to each variable $x, y, z, w$ for each $t \in[0,1]$, and there exist $u_{0}, w_{0}$ satisfying $T u_{0} \geq u_{0}, T w_{0} \leq w_{0}$ for $0 \leq u_{0} \leq w_{0}, 0 \leq u_{0}^{\prime} \leq w_{0}^{\prime}, 0 \leq t \leq 1$. Then BVPs (1.1)-(1.2) have at least a pair of positive solution $\left(u^{*}, v^{*}\right)$ such that $u_{0}(t) \leq$ $u^{*}(t) \leq w_{0}(t), v^{*}(t)=\int_{0}^{1} G_{\beta}(t, s) g\left(s, u^{*}(s), u^{*}(s)\right) d s$.

Proof Define the normal cone $P \subset E$ as (3.2) and the operator $T: P \rightarrow P$ as (3.3). By the definition of $T$, it is easy to show that $T$ is continuous. For any bounded subset $\Omega=\{u \in P:\|u\|<R\}$ of $P$, similar to the proof of (3.6) in Theorem 3.1, we know that $T(\Omega) \subset \Omega \subset P$ which implies that $P$ is relatively compact set in $E$. Hence $T: P \rightarrow P$ is completely continuous. 
For any $\bar{u}, \bar{v} \in P$ defined by (3.2), we define the relationship $\leq$ on $P$ as $\bar{v} \leq \bar{u}$. It is easy to verify that $\leq$ is a partial order on $P$. Let $u, w \in P$ be such that $u \leq w, u^{\prime} \leq w^{\prime}$, then $g\left(t, u(t), u^{\prime}(t)\right) \leq g\left(t, w(t), w^{\prime}(t)\right)$, for $t \in[0,1]$. Thus we have

$$
\begin{aligned}
(T u)(t)= & \int_{0}^{1} G_{\alpha}(t, s) f\left(s, u(s), \int_{0}^{1} G_{\beta}(s, \tau) g\left(\tau, u(\tau), u^{\prime}(\tau)\right) d \tau, u^{\prime}(s),\right. \\
& \left.\int_{0}^{1} G_{\beta}^{\prime}(s, \tau) g\left(\tau, u(\tau), u^{\prime}(\tau)\right) d \tau\right) d s \\
\leq & \int_{0}^{1} G_{\alpha}(t, s) f\left(s, w(s), \int_{0}^{1} G_{\beta}(s, \tau) g\left(\tau, w(\tau), w^{\prime}(\tau)\right) d \tau, w^{\prime}(s),\right. \\
& \left.\int_{0}^{1} G_{\beta}^{\prime}(s, \tau) g\left(\tau, w(\tau), w^{\prime}(\tau)\right) d \tau\right) d s \\
= & (T w)(t) .
\end{aligned}
$$

Hence $T$ is an increasing operator. By the assumptions $T u_{0} \geq u_{0}, T w_{0} \leq w_{0}$, we have $T$ : $\left\langle u_{0}, w_{0}\right\rangle \rightarrow\left\langle u_{0}, w_{0}\right\rangle$. Since $T: P \rightarrow P$ is completely continuous, by Lemma $2.3, T$ has one fixed point $u^{*} \in\left\langle u_{0}, w_{0}\right\rangle$. Thus BVPs (1.1)-(1.2) have at least a pair of positive solution $\left(u^{*}, v^{*}\right)$ such that $u_{0}(t) \leq u^{*}(t) \leq w_{0}(t), v^{*}(t)=\int_{0}^{1} G_{\beta}(t, s) g\left(s, u^{*}(s), u^{*}(s)\right) d s$. The proof is complete.

Theorem 3.6 Assume that $\left(\mathrm{H}_{1}\right)-\left(\mathrm{H}_{2}\right)$ hold. Assume $A_{1} f(t, x, y, z, w)<\frac{1}{2}(x+y+z+w)$ and $B_{1} g(t, x, y)<x+y$ for $t \in[0,1], 0 \leq x+y \leq x+y+z+w<\infty$. Then BVPs (1.1)-(1.2) have no monotone positive solution.

Proof Define the cone $P \in E$ as (3.2), the operator $T: P \rightarrow P$ as (3.3) and the partial order $\leq$ on $P$ as the proof of Theorem 3.5. By the definition of $T$, it is easy to show that $T$ is continuous. For any bounded subset $\Omega=\{u \in P:\|u\|<R\}$ of $P$, similar to the proof of (3.6) in Theorem 3.1, we know that $T(\Omega) \subset \Omega \subset P$, which implies that $P$ is relatively compact set in $E$. Hence $T: P \rightarrow P$ is completely continuous.

Suppose on the contrary that $u$ is a monotone positive solution of BVPs (1.1)-(1.2). Then, for $t \in[0,1]$, we obtain $u(t) \geq 0, u^{\prime}(t) \geq 0$, and

$$
\begin{aligned}
\|u\|= & \max _{0 \leq t \leq 1}|u(t)|+\max _{0 \leq t \leq 1}\left|u^{\prime}(t)\right| \\
= & \int_{0}^{1}\left[G_{\alpha}(t, s)+G_{\alpha}^{\prime}(t, s)\right] f\left(s, u(s), \int_{0}^{1} G_{\beta}(s, \tau) g\left(\tau, u(\tau), u^{\prime}(\tau)\right) d \tau, u^{\prime}(s),\right. \\
& \left.\int_{0}^{1} G_{\beta}^{\prime}(s, \tau) g\left(\tau, u(\tau), u^{\prime}(\tau)\right) d \tau\right) d s \\
< & \frac{1}{2 A_{1}} \int_{0}^{1}\left(J_{\alpha}(s)+\frac{\alpha-1}{\alpha-2} J_{\alpha}(s)\right)\left(\int_{0}^{1}\left[G_{\beta}(s, \tau)+G_{\beta}^{\prime}(s, \tau)\right]\right. \\
& \left.\times g\left(\tau, u(\tau), u^{\prime}(\tau)\right) d \tau+u(s)+u^{\prime}(s)\right) d s \\
< & \frac{1}{2 A_{1}} \frac{2 \alpha-3}{\alpha-2} \int_{0}^{1} J_{\alpha}(s) d s\left[\frac{1}{B_{1}} \int_{0}^{1}\left(J_{\beta}(\tau)+\frac{\beta-1}{\beta-2} J_{\beta}(\tau)\right)\left[u(\tau)+u^{\prime}(\tau)\right] d \tau+\|u\|\right] \\
< & \frac{1}{2 A_{1}} \frac{2 \alpha-3}{\alpha-2} \int_{0}^{1} J_{\alpha}(s) d s\left[\frac{1}{B_{1}} \frac{2 \beta-3}{\beta-2} \int_{0}^{1} J_{\beta}(\tau) d \tau+1\right]\|u\|<\|u\|,
\end{aligned}
$$


which is a contradiction. Then BVPs (1.1)-(1.2) have no monotone positive solution. The proof is complete.

Similarly, we obtain the following theorem.

Theorem 3.7 Assume that $\left(\mathrm{H}_{1}\right)-\left(\mathrm{H}_{2}\right)$ hold. If $A_{2} f(t, x, y, z, w)>\frac{1}{2}(x+y+z+w)$ and $B_{2} g(t, x, y)>x+y$ for $t \in[\theta, 1-\theta], 0 \leq x+y \leq x+y+z+w<\infty$. Then BVPs (1.1)-(1.2) have no monotone positive solution.

\section{Illustrative examples}

Consider the following coupling system of fractional differential equations:

$$
\begin{cases}D_{0+}^{\frac{5}{2}} u(t)+f\left(t, u(t), v(t), u^{\prime}(t), v^{\prime}(t)\right)=0, & t \in(0,1), n=3, \\ D_{0+}^{\frac{7}{2}} v(t)+g\left(t, u(t), u^{\prime}(t)\right)=0, & t \in(0,1), m=4,\end{cases}
$$

subject to the integral boundary conditions

$$
\left\{\begin{array}{l}
u(0)=u^{\prime \prime}(0)=0, \quad u^{\prime}(1)=\int_{0}^{1} u(s) d H(s), \\
v(0)=v^{\prime \prime}(0)=v^{\prime \prime \prime}(0)=0, \quad v^{\prime}(1)=\int_{0}^{1} v(s) d K(s),
\end{array}\right.
$$

where $H(t)=t^{2}, K(t)=t^{3}$ for all $t \in[0,1]$. Then we obtain

$$
\begin{aligned}
& \Delta_{1}=1-\int_{0}^{1} s d H(s)=1-2 \int_{0}^{1} s^{2} d s=\frac{1}{3}>0, \\
& \Delta_{2}=1-\int_{0}^{1} s d K(s)=1-3 \int_{0}^{1} s^{3} d s=\frac{1}{4}>0 .
\end{aligned}
$$

Take $\theta=\frac{1}{4}$, for the functions $J_{\alpha}$ and $J_{\beta}$, we obtain

$$
\begin{aligned}
J_{\alpha}(s)= & g_{\alpha}(1, s)+\frac{1}{\Delta_{1}} \int_{0}^{1} g_{\alpha}(\tau, s) d H(\tau) \\
= & \frac{1}{\Gamma(\alpha)}\left\{(\alpha-1)(1-s)^{\alpha-2}-(1-s)^{\alpha-1}\right. \\
& \left.+6\left(\int_{0}^{1}(\alpha-1) \tau^{2}(1-s)^{\alpha-2} d \tau-\int_{s}^{1} \tau(\tau-s)^{\alpha-1} d \tau\right)\right\} \\
= & \frac{4}{3 \sqrt{\pi}}\left[\frac{9}{2}(1-s)^{\frac{1}{2}}-(1-s)^{\frac{3}{2}}-\frac{24 s+60}{35}(1-s)^{\frac{5}{2}}\right], \quad s \in[0,1],
\end{aligned}
$$

and

$$
\begin{aligned}
J_{\beta}(s)= & g_{\beta}(1, s)+\frac{1}{\Delta_{2}} \int_{0}^{1} g_{\beta}(\tau, s) d K(\tau) \\
= & \frac{1}{\Gamma(\beta)}\left\{(\beta-1)(1-s)^{\beta-2}-(1-s)^{\beta-1}\right. \\
& \left.+12\left(\int_{0}^{1}(\beta-1) \tau^{3}(1-s)^{\beta-2} d \tau-\int_{s}^{1} \tau^{2}(\tau-s)^{\beta-1} d \tau\right)\right\} \\
= & \frac{8}{15 \sqrt{\pi}}\left[10(1-s)^{\frac{3}{2}}-(1-s)^{\frac{5}{2}}-\frac{24\left(8 s^{2}+28 s+63\right)}{693}(1-s)^{\frac{7}{2}}\right], \quad s \in[0,1] .
\end{aligned}
$$


A simple calculation shows that

$$
\begin{array}{ll}
A_{1}=\frac{2 \alpha-3}{\alpha-2} \int_{0}^{1} J_{\alpha}(s) d s \approx 6.2186, & A_{2}=\left(\theta^{\alpha-1}+\gamma_{1}\right) \int_{\theta}^{1-\theta} J_{\alpha}(s) d s \approx 0.3075, \\
B_{1}=\frac{2 \beta-3}{\beta-2} \int_{0}^{1} J_{\beta}(s) d s \approx 2.5571, & B_{2}=\gamma_{0}\left(\theta^{\beta-1}+\gamma_{1}^{\prime}\right) \int_{\theta}^{1-\theta} J_{\beta}(s) d s \approx 0.0080 .
\end{array}
$$

Case 1. Let

$$
\begin{aligned}
& f\left(t, u, v, u^{\prime}, v^{\prime}\right)=\frac{1}{16(1+t)}\left[\frac{u+v+u^{\prime}+v^{\prime}}{e^{u+v+u^{\prime}+v^{\prime}}}+\frac{50\left(u+v+u^{\prime}+v^{\prime}\right)^{2}}{1+u+v+u^{\prime}+v^{\prime}}\right], \\
& g\left(t, u, u^{\prime}\right)=\frac{1}{1+t}\left[\frac{u+u^{\prime}}{4 \ln \left[e+u+u^{\prime}\right]}+\frac{250\left(u+u^{\prime}\right)^{2}}{1+u+u^{\prime}}\right] .
\end{aligned}
$$

Clearly, $f(t, 0,0,0,0)=g(t, 0,0)=0$. By a simple computation, we get $f^{0}=\frac{1}{16}, f_{\infty}=\frac{25}{14}$, $g^{0}=\frac{1}{4}$, and $g_{\infty}=\frac{1,000}{7}$, which implies that $A_{1} f^{0} \approx 0.38866<\frac{1}{2}<0.54911 \approx A_{2} f_{\infty}$ and $B_{1} g^{0} \approx 0.63928<1<1.14286 \approx B_{2} g_{\infty}$. Hence BVPs (4.1)-(4.2) have at least a pair of positive solutions by Theorem 3.1.

Case 2. Let

$$
\begin{aligned}
& f\left(t, u, v, u^{\prime}, v^{\prime}\right)=\frac{7}{1+t}\left[\frac{u+v+u^{\prime}+v^{\prime}}{e^{u+v+u^{\prime}+v^{\prime}}}+\frac{\left(u+v+u^{\prime}+v^{\prime}\right)^{2}}{10^{5}+u+v++u^{\prime}+v^{\prime}}\right], \\
& g\left(t, u, u^{\prime}\right)=\frac{240}{1+t}\left[\frac{u+u^{\prime}}{e^{u+u^{\prime}}}+\frac{\left(u+u^{\prime}\right)^{2}}{10^{6}+u+u^{\prime}}\right] .
\end{aligned}
$$

Clearly, $f(t, 0,0,0,0)=g(t, 0,0)=0$. By a simple computation, we obtain $f_{0}=f_{\infty}=4$, $g_{0}=g_{\infty}=\frac{960}{7}$, and $g^{0}=240$, which shows that $A_{2} f_{0} \approx 1.23>\frac{1}{2}, A_{2} f_{\infty} \approx 1.23>\frac{1}{2}, B_{2} g_{0} \approx$ $1.09714>1, B_{2} g_{\infty} \approx 1.09714>1$, and $B_{2} g^{0} \approx 1.92<2$.

Choose $\mu=10^{3}$, we get

$$
\begin{gathered}
\max \left\{g(t, x, y): t \in[0,1], x+y \in\left[0,10^{3}\right]\right\}<240\left(\frac{1}{e}+\frac{10^{6}}{10^{6}+10^{3}}\right) \approx 328.0513 \\
<\frac{\mu}{B_{1}} \approx 391.0680 \\
\max \left\{f(t, x, y, z, w): t \in[0,1], x+y+z+w \in\left[0,10^{3}\right]\right\}<7\left(\frac{1}{e}+\frac{10^{6}}{10^{5}+10^{3}}\right) \approx 71.8821 \\
<\frac{\mu}{A_{1}} \approx 160.8079 .
\end{gathered}
$$

Hence BVPs (4.1)-(4.2) have at least two pairs of positive solutions by Theorem 3.3.

Competing interests

The authors declare that they have no competing interests.

Authors' contributions

All authors contributed equally and significantly in writing this paper. All authors read and approved the final manuscript.

\section{Acknowledgements}

The author would like to thank the anonymous referees for their useful and valuable suggestions. This work is supported by the National Natural Sciences Foundation of Peoples Republic of China under Grant (No. 11161025), Yunnan Province natural scientific research fund project (No. 2011FZ058). 


\section{References}

1. Lakshmikantham, V, Leela, S: Nagumo-type uniqueness result for fractional differential equations. Nonlinear Anal. 71, 2886-2889 (2009)

2. Chen, F, Zhou, Y: Attractivity of fractional functional differential equations. Comput. Math. Appl. 62, 1359-1369 (2011)

3. Chang, Y, Nieto, J: Some new existence results for fractional differential inclusions with boundary conditions. Math. Comput. Model. 49, 605-609 (2009)

4. Kilbas, AA, Trujillo, Jj: Differential equations of fractional order: methods, results and problems-I. Appl. Anal. 78, 153-192 (2001)

5. Kilbas, AA, Trujillo, JJ: Differential equations of fractional order: methods, results and problems-II. Appl. Anal. 81, 435-493 (2002)

6. Bai, Z: On positive solutions of a nonlocal fractional boundary value problem. Nonlinear Anal. 72, 916-924 (2010)

7. Bai, Z, Qiu, T: Existence of positive solution for singular fractional differential equation. Appl. Math. Comput. 215(7), 2761-2767 (2009)

8. Ahmad, B, Nieto, JJ: Existence results for a coupled system of nonlinear fractional differential equations with three-point boundary conditions. Comput. Math. Appl. 58(9), 1838-1843 (2009)

9. Henderson, J, Luca, R: Existence and multiplicity of positive solutions for a system of fractional boundary value problems. Bound. Value Probl. 2014, Article ID 60 (2014)

10. Ahmad, B, Ntouyas, S, Alsaedi, A: New existence results for nonlinear fractional differential equations with three-point integral boundary conditions. Adv. Differ. Equ. 2011, Article ID 107384 (2011)

11. Webb, JRL: Positive solutions of some higher order nonlocal boundary value problems. Electron. J. Qual. Theory Differ. Equ. 2009, 29 (2009)

12. Webb, JRL, Infante, G: Nonlocal boundary value problems of arbitrary order. J. Lond. Math. Soc. 79(2), 238-258 (2009)

13. Feng, $M, J i, D, G e, W$ : Positive solutions for a class of boundary-value problem with integral boundary conditions in Banach spaces. J. Comput. Appl. Math. 222, 351-363 (2008)

14. Jia, M, Liu, X: Three nonnegative solutions for fractional differential equations with integral boundary conditions. Comput. Math. Appl. 62, 1405-1412 (2011)

15. Zhang, HE: Multiple positive solutions of nonlinear BVPs for differential systems involving integral conditions. Bound. Value Probl. 2014, Article ID 61 (2014)

16. Chatthai, T, Jessada, T, Sotiris, KN: Impulsive fractional boundary-value problems with fractional integral jump conditions. Bound. Value Probl. 2014, Article ID 17 (2014)

17. Kilbas, A, Srivastava, H, Trujillo, J: Theory and Applications of Fractional Differential Equations. North-Holland Mathematics Studies, vol. 204. Elsevier, Amsterdam (2006)

18. Podlubny, I: Fractional Differential Equations. Academic Press, New York (1993)

19. Guo, D, Lakshmikantham, V, Liu, X: Nonlinear Integral Equations in Abstract Spaces. Mathematics and Its Applications, vol. 373. Kluwer Academic, Dordrecht (1996)

20. Joshi, MC, Bose, RK: Some Topics in Nonlinear Functional Analysis. Wiley Eastern, New Delhi (1985)

10.1186/1687-1847-2014-254

Cite this article as: Zhao and Gong: Positive solutions of Riemann-Stieltjes integral boundary problems for the nonlinear coupling system involving fractional-order differential. Advances in Difference Equations 2014, 2014:254

\section{Submit your manuscript to a SpringerOpen ${ }^{\circledR}$ journal and benefit from:}

- Convenient online submission

- Rigorous peer review

Immediate publication on acceptance

- Open access: articles freely available online

- High visibility within the field

- Retaining the copyright to your article 